\title{
Control-display alignment determines the prevalent compatibility effect in two-dimensional stimulus-response tasks
}

\author{
Samuel Lee ${ }^{1}$ James D. Miles ${ }^{1,2} \cdot$ Kim-Phuong L. Vu ${ }^{1}$
}

Published online: 14 July 2015

(C) Psychonomic Society, Inc. 2015

\begin{abstract}
Responses are faster and more accurate when they are spatially compatible with a stimulus than when they are incompatible (the stimulus-response compatibility (SRC) effect). In studies using two-dimensional (2-D) stimulus and response sets in which stimuli and responses have both vertical (top-bottom) and horizontal (right-left) spatial relations, SRC effects are generally larger along the horizontal dimension, an effect called right-left prevalence. Several accounts have been posited to explain this asymmetry, including frames of reference to the body and spatio-anatomical constraints. We propose a new account of the right-left prevalence effect in which prevalence effects are largely determined by the spatial alignment between elements on the stimulus display and response locations on the control panel-the control-display alignment (CDA). For example, when responses are aligned below a display, 2-D stimulus and response sets share a common vertical midline that emphasizes a right-left distinction. When responses are to the right or left of the display, the shared midline is horizontal, emphasizing the top-bottom distinction and should instead lead to top-bottom prevalence effects. Participants completed two-choice, 2-D SRC tasks in four control-display configurations with a response panel centered above, below, left, and right of a projected display. As hypothesized, right-left prevalence was elicited using vertical CDA and top-bottom prevalence was elicited using horizontal CDA. The findings demonstrate that CDA largely determines
\end{abstract}

James D. Miles

jmiles@csulb.edu

1 California State University Long Beach, Long Beach, CA, USA

2 Psychology Department, California State University, $1250 \mathrm{~N}$ Bellflower Blvd., Long Beach, CA 90840, USA prevalence effects and should be taken into account when using multidimensional stimulus and response sets.

Keywords Stimulus-response compatibility $\cdot$ Motor planning/programming $\cdot$ Spatial cognition

The stimulus-response compatibility (SRC) effect refers to the fact that people respond faster and more accurately when responses are spatially compatible with stimulus position than when they are not (for review see Proctor \& Reeve, 1990). SRC effect magnitude (incompatible mapping minus compatible mapping) is generally largest when the possible task responses (i.e., the response set) share many features with the stimulus set, also known as dimensional overlap (Kornblum, Hasbroucq, \& Osman, 1990).

Separable SRC effects occur when stimulus and response sets are arranged two-dimensionally (2-D). For example, stimuli arranged in the top-left and bottom-right of a display and responses arranged in the top-right and bottom-left of a control panel may have a stimulus-response mapping that is compatible on the top-bottom dimension but not the right-left dimension, or vice versa. Such 2-D arrangements consistently find that compatibility effects for the right-left dimension are greater than for the top-bottom dimension - an effect known as the right-left prevalence effect (Nicoletti \& Umiltà, 1984, 1985). Nicoletti et al. (1982) initially suggested that right-left prevalence occurs because the left and right hands often are used as response effectors. This provides a spatio-anatomical reference frame for coding all responses along the right-left dimension. However, evidence for this view is mixed: no right-left prevalence effect occurs when the right and left fingers on the same hand were used for responding (Vu \& Proctor, 2001) even though fingers can be coded as left and right effectors (Heister, Schroeder-Heister, \& Ehrenstein, 1990). A right-left 
prevalence effect also is found when the effector distinction emphasizes the top-bottom dimension (left hand and right foot; Nicoletti and Umiltà (1985). However, a top-bottom prevalence effect was found when ipsilateral effectors (i.e., left hand and left foot) were used for responding (Vu \& Proctor, 2001; Chan \& Chan, 2011). When only a single effector was used to respond (unimanual joystick responses), no right-left prevalence effect occurred, indicating that the spatial effector distinction may be required to obtain the effect (Hommel, 1996).

The right-left prevalence effect may also be caused by the human body's right-left midline barrier (Nicoletti \& Umiltà, 1985). In this case, body midline serves as a prominent reference axis for coding horizontal spatial locations and right-left prevalence effects result from the use of an egocentric, or body-based, spatial frame of reference regardless of the actual effectors used (Nicoletti, Umiltà, Tressoldi, \& Marzi, 1988). In contrast with this view, when a vertical frame of reference between the body and stimulus positions is induced by aligning the display-control configuration relative to the participants' eye level (stimuli above and below the eyes), rightleft prevalence effects still occur (Hommel, 1996)

Right-left prevalence effects also may reflect the way that people conceptualize the rules of the task, which commonly include instructions that emphasize the right-left relation between stimulus and response sets (Ansorge \& Wuhr, 2004; Hommel, 1993, 1996). When instructions emphasize topbottom rather than right-left stimulus-response relations, the magnitude of the right-left prevalence effect is reduced but not fully eliminated, suggesting that instructions moderate the prevalence effect but are not the only cause.

The mixed evidence for these accounts may indicate that effector, body midline, and instruction all contribute to the prevalence effect indirectly by altering which response dimension was most salient (Vu \& Proctor, 2001). In fact, when the salient response dimension is manipulated by varying the spacing between stimuli, whether hands were crossed or uncrossed, and task instructions, each contributed to the direction of the prevalence effect (Vu \& Proctor, 2002).

\section{Control-display alignment}

We suggest that there is another reason for commonly observed right-left prevalence effects - the spatial relation between the location of the display and the control panel. We refer to this as the control-display alignment (CDA). CDA is allocentric in nature - the prevalent stimulus-response dimension changes depending on the location of response positions in relation to the stimulus display. In previous studies of right-left prevalence, the control panel was commonly positioned below the display (either on a table for hand responses or on the floor for foot responses) leading to a vertical CDA (Nicoletti \& Umiltà, 1985; Rubichi, Nicoletti, Pelosi, \& Umiltà, 2004; Vu,
Pellicano, \& Proctor, 2005). As shown in Fig. 1, with a vertical CDA, stimulus and response sets share an absolute right-left distinction in space - both are bisected by a single vertical midline. This increases the apparent dimensional overlap between the right and left stimulus and response positions, leading to a prevalent right-left compatibility effect. Conversely, if the control panel is positioned to the left or right side of the stimulus display (horizontal CDA), the shared midline bisects the stimulus and response sets horizontally, making the topbottom dimensional overlap more salient and leading to prevalent top-bottom compatibility effects. In other words, the dominant spatial dimension in multidimensional stimulusresponse sets is the result of dimensional salience cause by CDA. Previous research has shown that the magnitude of unidimensional SRC effects is influenced by similar environmental frames of reference under certain circumstances (Ladavas \& Moscovitch, 1984; Lamberts, Tavernier, \& d'Ydewalle, 1992; Roswarski \& Proctor, 1996). However, no prior work has examined whether changing the spatial alignment between the display and control panel directly determines the prevalent spatial dimension when utilizing 2-D stimulus-response sets.

Based on the CDA account, we predict that when a control panel is below or above a display, the right-left compatibility effect will be more pronounced than top-bottom compatibility effect, leading to right-left prevalence similar to previous studies. When the control panel is aligned horizontally with the display (to the left or right), the top-bottom compatibility effect will be more pronounced than the right-left compatibility

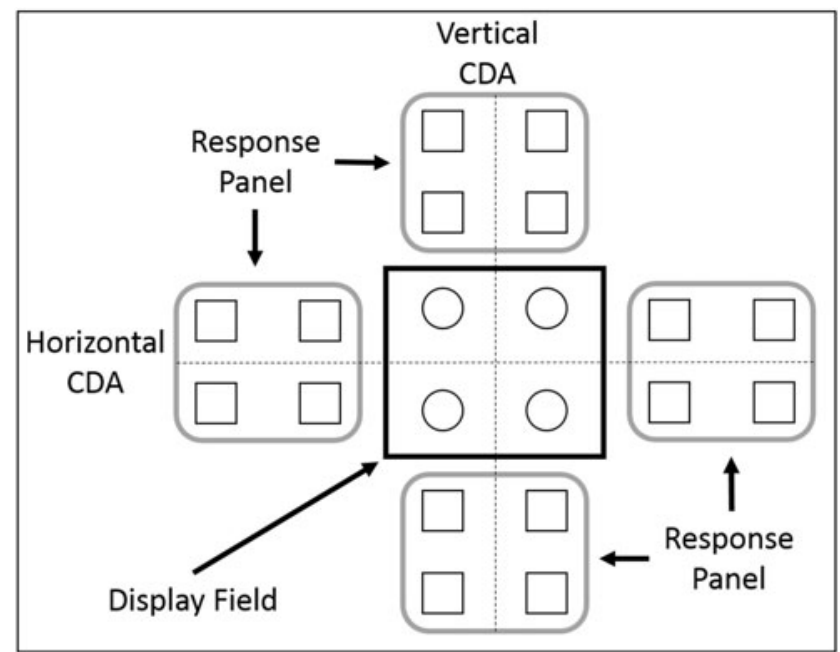

Fig. 1 The arrangement of the stimulus display field (center frame with circle targets inside) and each of the possible control panel locations (grey frames with square buttons inside), which were presented on a horizontal table top. The control panel was either above or below (vertical CDA) or to the left or right (horizontal CDA) in relation to the display. Dotted lines represent the midline barrier between the stimuli on the display and the responses on the control panel. With horizontal CDA, the shared midline of the stimuli and response sets divides them into top and bottom regions and with vertical CDA, their shared midline divides them into left and right regions 
effect, producing top-bottom prevalence. This is a unique prediction of the CDA account - accounts based on response effectors or egocentric body space predict no difference between vertical and horizontal CDA, because there are no changes in the effectors or spatial frame of reference between the response positions and body.

As aforementioned, the dimension emphasized in the task instructions also may affect prevalence effects (Hommel, 1996) and interact with other factors influencing prevalence effects (Vu \& Proctor, 2001). We therefore also included an instruction manipulation emphasizing responses along either the right-left or top-bottom dimension. An interactive effect of CDA and instruction type on the prevalence effect would indicate that CDA likely influences the prevalence effect by influencing how participants conceptualize the task. Additive influences of instruction and CDA would instead indicate that CDA is a unique influence on prevalence effects.

\section{Method}

\section{Participants}

Sixty-four participants (34 females and 30 males; age range: 18 -29 yr $(M=21.31, S D=3.23))$ were recruited from our Psychology Department's subject pool. All participants reported having normal or corrected-to-normal vision. Participants received 1 hour of experimental credit for their time.

\section{Apparatus and stimuli}

The experiment program was written using Visual Basic and presented using a Windows 7 PC. A digital light processing projector displayed the stimuli on a vertical tabletop covered with a non-reflective white surface. A $20-\mathrm{cm}$ x $20-\mathrm{cm}$ stimulus display field with a white frame was projected on the table approximately $40 \mathrm{~cm}$ away from the participant while seated in front of the projector table. On each trial, a 1.9-cm, white cross $\left(3^{\circ}\right.$ visual angle) was projected at the center of the display field, which served as the fixation point prior to each target stimulus. The target stimulus was a filled white circle $1.9 \mathrm{~cm}$ in diameter, presented in either the upper-left, upperright, bottom-left, or bottom-right quadrant of the display field with the center of the target $9.5 \mathrm{~cm}$ from the center of the fixation cross. An Ergodex DX1 control panel (30-cm wide $\times 24-\mathrm{cm}$ long) was positioned either above or below (vertical CDA) or to the right or left (horizontal CDA) of the display field in separate trial blocks (grey boxes represent different control panel positions in Fig. 1). The control panel had four response buttons arranged in a similar fashion to the targets on the display field (upper-left, upper-right, bottom-left, bottomright) and were each $9.5 \mathrm{~cm}$ away from the center of the control panel. In each control panel position, the control panel was positioned such that the center point of its four keys was $25 \mathrm{~cm}$ away from the center of the display field.

\section{Design and procedure}

On any trial block, only two diagonal target positions and two diagonal response buttons were used: target circles were either only in the upper-left and bottom-right position or only in the upper-right and bottom-left positions. The unused target positions did not appear on the display. Response positions on the control panel were arranged in the same way. This led to four configurations of stimulus and response positions (Fig. 2). For each of these configurations, participants performed separate trial blocks for both of the possible mappings between the stimulus and response positions, leading to a total of eight trial blocks that represented all combinations of right-left and topbottom compatibility equally. Each block contained 8 practice and 20 test trials, with an equal number of trials at the 2 target positions presented in a pseudorandom order. Each of these types of trial blocks was performed at all 4 control panel positions, leading to a total of 32 blocks. Note that Fig. 2 only shows these block types when the control panel is below the display field; however, the combinations of right-left and topbottom compatibilities remained invariant across control panel position.

The experiment was conducted in a quiet, dimly lit room. Participants were seated in front of the projection table so that they were centered with the control panel. The left index finger was placed on the left button and the right index finger on the right button. Before every block of trials, instructions appeared at the center of the display field indicating the correct response button for each of the two target positions. The instructions only provided the stimulus-response mapping along a single dimension. Half the participants always received right-left instructions on the display field and the other half always received top-bottom instructions. In the Right-Left Instructions condition, top-left and bottom-left targets were referred to as the "left circle" and the top-right and bottom-right targets were referred to as the "right circle." The corresponding response buttons were referred to in a similar fashion using the same spatial dimension as the targets. For example, if right-left instructions were provided, the participant was told on compatible trial blocks that if a left circle appears, make a left response and if a right circle appears, make a right button response. For incompatible trial blocks, participants were told that if the left circle appears, make a right response and if right circle appears, make a left response. Alternatively, participants receiving the top-bottom instructions only saw the words "top" and "bottom" to refer to locations of target circles and responses in both the compatible and incompatible trial blocks.

Each trial commenced with a 1000-ms blank screen followed by the display fixation cross for $500 \mathrm{~ms}$. After the fixation 


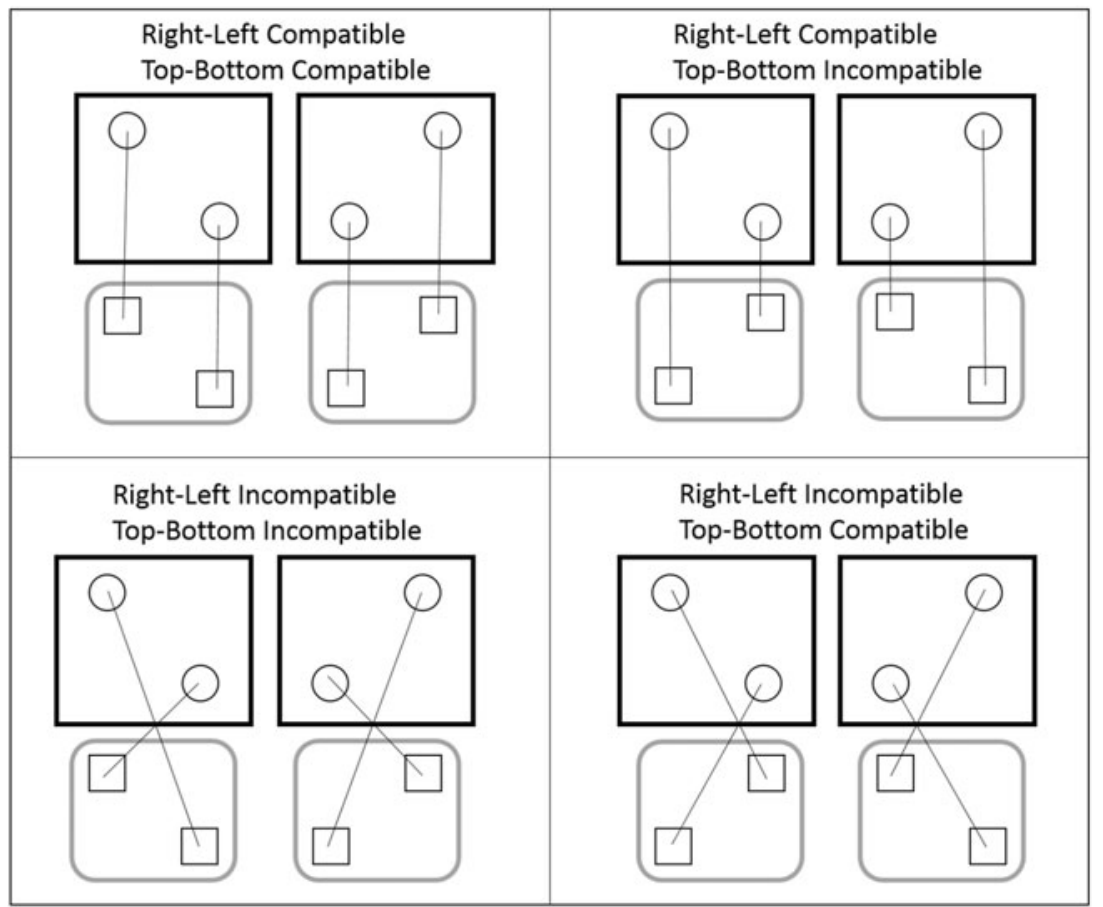

Fig. 2 All eight possible combinations of targets, responses, and mappings between them when the control panel (grey frame with square buttons) is below the display field (black frame with circle targets). These combinations and their resulting right-left and topbottom compatibilities are the same for all control panel positions disappeared, the target appeared in one of the two possible diagonal corners indicated for that trial block and was displayed until the participant responded by pressing the button associated with the target position in the instructions. Response times (RTs) were measured as the time elapsed between target onset and the key press. If a correct response was given, the screen blanked for $1000 \mathrm{~ms}$ and the fixation cross reappeared for $500 \mathrm{~ms}$, prompting the start of a new trial. If an incorrect response was given, a 300-ms auditory bell was presented through computer speakers to alert the participant, after which the normal sequence for a correct response was resumed. This was repeated at the same control panel position for each of the 8 trial block types. The researcher then moved the control panel to the next position and repeated each of the block types. Response panel position and trial type orders were randomly assigned using a partial Latin square. In total, each participant completed 32 blocks for a total of 640 experimental trials and 256 practice trials, which took approximately 1 hour.

\section{Results}

RTs below $100 \mathrm{~ms}$ (anticipations) and RTs greater than $2500 \mathrm{~ms}$ were considered outliers and not included $(<1 \%$ of responses). Trials in which the control panel was above or below the stimulus display were combined to form the vertical
CDA condition and trials with the control panel to the left or right formed the horizontal CDA condition. Mean RTs and mean percent error (PE) were submitted to separate 2 (CDA: vertical CDA or horizontal CDA) x 2 (Right-Left Compatibility: compatible or incompatible) x 2 (Top-Bottom Compatibility: compatible or incompatible) x 2 (Instruction: topbottom or right-left) mixed analyses of variance. CDA, Right-Left Compatibility, and Top-Bottom Compatibility were within-participant factors and Instruction was a between-groups factor. Alpha was set at $p<0.05$ for statistical significance in all analyses. Response Times (RT) and Percent Errors (PE) for all conditions are shown in Table 1.

\section{Response time}

Error trials were not included in the RT analysis ( $2 \%$ of responses). The main effect of CDA was significant, $F(1,62)=$ $22.50, p<0.05, \eta p^{2}=0.27$, such that RTs with vertical CDAs $(M=398 \mathrm{~ms})$ were $16 \mathrm{~ms}$ faster than RTs with horizontal CDAs $(M=414 \mathrm{~ms})$. Participants also were faster on rightleft compatible $(M=388 \mathrm{~ms})$ compared to right-left incompatible $(M=424 \mathrm{~ms})$ trials, $F(1,62)=122.07, p<0.05, \eta p^{2}=$ 0.66 , and faster on top-bottom compatible $(M=387 \mathrm{~ms})$ than top-bottom incompatible $(M=426 \mathrm{~ms})$ trials, $F(1,62)=$ 100.98, $p<0.05, \eta p^{2}=0.62$.

As predicted by the CDA account of prevalence effects, the right-left compatibility effect was larger with a vertical 
Table 1 Mean RT (ms) and PE (\%) as a function of right-left compatibility, top-bottom compatibility, instructions, and CDA (standard error in parentheses). Compatibility Effect $=$ Incompatible RT - Compatible RT

\begin{tabular}{|c|c|c|c|c|c|c|}
\hline \multirow[b]{3}{*}{ Right-Left Compatibility } & \multicolumn{6}{|c|}{ Top-Bottom Compatibility } \\
\hline & \multicolumn{2}{|c|}{ Compatible } & \multicolumn{2}{|c|}{ Incompatible } & \multicolumn{2}{|c|}{ Compatibility Effect } \\
\hline & RT & $\mathrm{PE}$ & RT & $\mathrm{PE}$ & RT & PE \\
\hline & \multicolumn{6}{|c|}{ Right-Left Instructions } \\
\hline \multicolumn{7}{|l|}{ Horizontal CDA } \\
\hline Compatible & $362(8)$ & $.7(.2)$ & $422(15)$ & $2.8(.5)$ & 60 & 2.1 \\
\hline Incompatible & $416(9)$ & $3.5(.5)$ & $438(13)$ & $3.4(.6)$ & 12 & -0.1 \\
\hline Compatibility Effect & 54 & 2.8 & 16 & 0.6 & & \\
\hline \multicolumn{7}{|l|}{ Vertical CDA } \\
\hline Compatible & $352(8)$ & $.7(.2)$ & $370(12)$ & $1.3(.3)$ & 18 & 0.6 \\
\hline Incompatible & $430(9)$ & $3.8(.5)$ & $420(10)$ & $3.2(.5)$ & -10 & -0.6 \\
\hline \multirow[t]{2}{*}{ Compatibility Effect } & 78 & 3.1 & 50 & 1.9 & & \\
\hline & \multicolumn{6}{|c|}{ Top-Bottom Instructions } \\
\hline \multicolumn{7}{|l|}{ Horizontal CDA } \\
\hline Compatible & $361(8)$ & $.7(.2)$ & $477(15)$ & $3.3(.5)$ & 116 & 2.6 \\
\hline Incompatible & $396(9)$ & $2.3(.5)$ & $438(13)$ & $2.3(.6)$ & 42 & 0 \\
\hline Compatibility Effect & 35 & 1.6 & -39 & -1 & & \\
\hline \multicolumn{7}{|l|}{ Vertical CDA } \\
\hline Compatible & $357(8)$ & $.7(.2)$ & $406(12)$ & $1.4(.3)$ & 49 & 0.7 \\
\hline Incompatible & $418(9)$ & $2.4(.5)$ & $435(10)$ & $2.6(.5)$ & 17 & 0.2 \\
\hline Compatibility Effect & 61 & 1.7 & 29 & 1.2 & & \\
\hline
\end{tabular}

$\mathrm{CDA}(M=55 \mathrm{~ms})$ than with a horizontal $\mathrm{CDA}(M=$ $16 \mathrm{~ms}), F(1,62)=41.72, p<0.05, \eta_{\mathrm{p}}{ }^{2}=0.40$. Conversely, the top-bottom compatibility effect was larger with a horizontal CDA $(M=60 \mathrm{~ms})$ than a vertical CDA $(M=$ $19 \mathrm{~ms}), F(1,62)=42.60, p<0.05, \eta_{\mathrm{p}}^{2}=0.41$ (Fig. 3). Paired sample $t$ tests confirmed that with the vertical CDA, the rightleft compatibility effect was significantly larger than the topbottom compatibility effect, $t(63)=4.93, p<0.05$, and the top-bottom compatibility effect was significantly larger than the right-left compatibility effect with the horizontal CDA, $t(63)=4.75, p<0.05$.

Right-Left Compatibility and Top-Bottom Compatibility also interacted with one another such that the right-left compatibility effect (Right-Left Incompatible RT minus RightLeft Incompatible RT) was largest when the top-bottom dimension was compatible and the top-bottom compatibility effect (Top-Bottom Incompatible RT minus Top-Bottom Incompatible RT) was largest when the right-left dimension was compatible, $F(1,62)=82.02, p<0.05, \eta_{\mathrm{p}}^{2}=0.57$. This increase in the compatibility effect in one dimension when the opposing dimension was compatible was greater with horizontal CDA than with vertical CDA, as indicated by a significant CDA x Right-Left Compatibility x Top-Bottom Compatibility interaction, $F(1,62)=9.24, p<0.05, \eta_{\mathrm{p}}{ }^{2}=0.13$.
Although there was no main effect of Instruction type ( $F<$ 1.0, $p>0.4$ ), Instruction type interacted with both Right-Left Compatibility and Top-Bottom Compatibility. As shown in Fig. 4, the top-bottom compatibility effect was greater with top-bottom instructions $(M=56 \mathrm{~ms})$ than with right-left instructions $(M=22 \mathrm{~ms}), F(1,62)=18.73, p<0.05, \eta_{\mathrm{p}}{ }^{2}=0.23$. Conversely, the right-left compatibility effect was greater with right-left instructions ( $\mathrm{M}=50 \mathrm{~ms})$ than with top-bottom instructions $(M=22 \mathrm{~ms}), F(1,62)=18.77, p<0.05, \eta_{\mathrm{p}}{ }^{2}=0.23$. As previously indicated, the compatibility effect in one dimension was reduced when the other dimension was incompatible; this reduction was greater with vertical instructions than with horizontal instructions, $F(1,62)=4.12, p<0.05, \eta_{\mathrm{p}}{ }^{2}=0.06$. The Instruction x CDA x Right-Left Compatibility x Top-Bottom Compatibility interaction was not significant, $F(1,62)=3.64, p$ $=0.06, \eta_{\mathrm{p}}{ }^{2}=0.06$, and no other interactions including Instruction and CDA reached significance (all $F \mathbf{s}<2.3$, all $p \mathrm{~s}>0.13$ ).

\section{Percent error}

PEs were low throughout the experiment (Table 1). Nonetheless, the data were consistent with the CDA account of prevalence effects. PE for vertical CDA $(\mathrm{M}=2.0 \%)$ was less than for horizontal CDA $(\mathrm{M}=2.4 \%), F(1,62)=4.01, p<0.05, \eta p^{2}=$ 


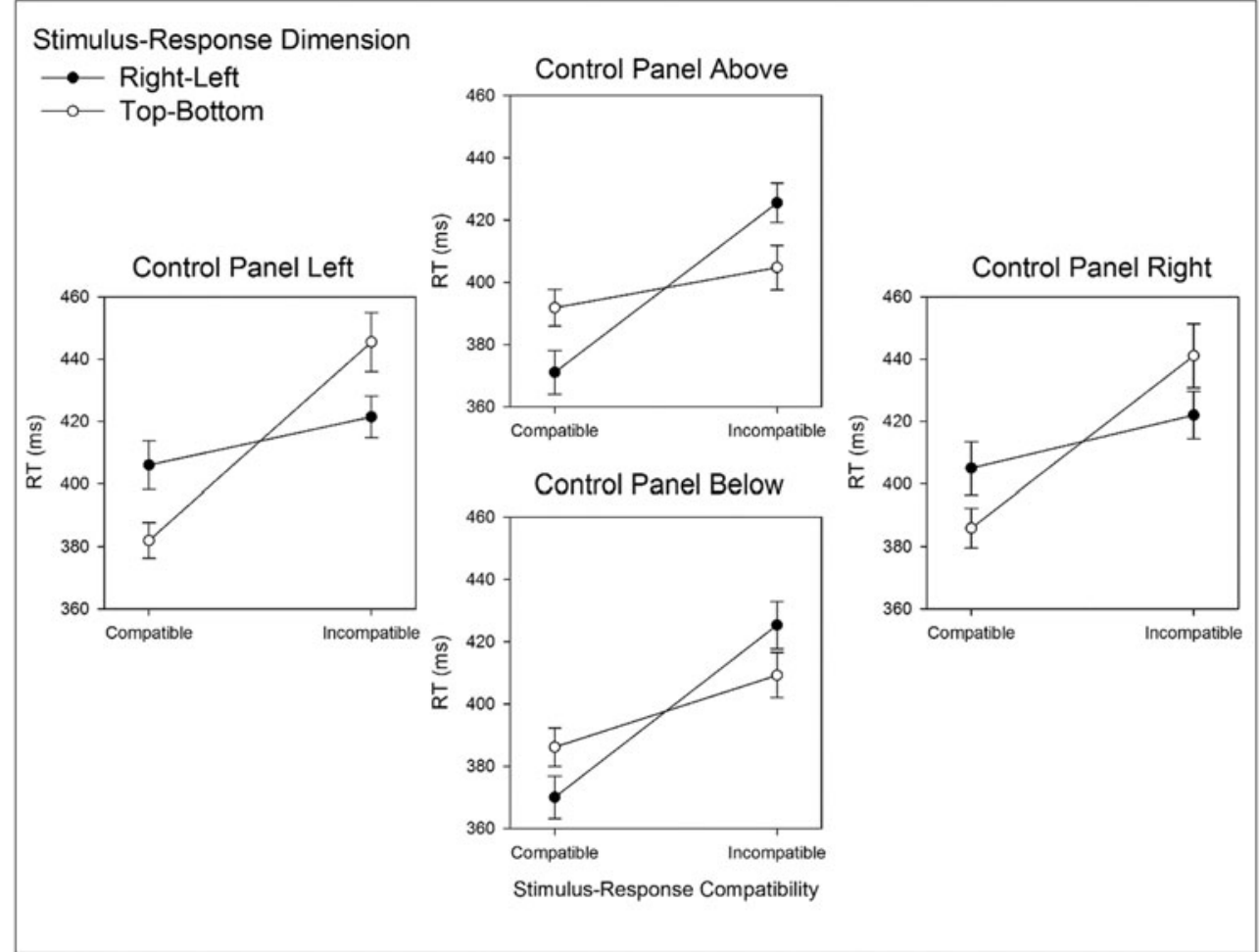

Fig. 3 RTs with standard errors for compatible and incompatible trials in the right-left dimension (filled circles) and the top-bottom dimension (open circles) of the two-dimensional stimulus and response set. Separate graphs represent data from each of the control panel positions

0.06. PEs were also lower for right-left compatible trials $(M=$ $1.4 \%)$ than right-left incompatible trials $(M=2.9 \%), F(1,62)=$ $45.30, p<0.05, \eta p^{2}=0.42$, and lower for top-bottom compatible trials $(M=1.8 \%)$ than top-bottom incompatible trials $(M=$ $2.5 \%), F(1,62)=11.08, p<0.05, \eta p^{2}=0.15$. The right-left compatibility effect for errors was greater with vertical CDA than horizontal CDA, $F(1,62)=10.63, p<0.05, \eta_{\mathrm{p}}{ }^{2}=0.15$, and the top-bottom compatibility effect was greater with horizontal CDA than vertical CDA, $F(1,62)=7.05, p<0.05, \eta_{\mathrm{p}}{ }^{2}=0.10$.

Compatibility effects were again greater for each dimension when the alternative dimension also was compatible, $F(1,62)=$ $21.28, p<0.05, \eta_{\mathrm{p}}{ }^{2}=0.26$, and this was more pronounced with a horizontal CDA than a vertical CDA $F(1,62)=5.35, p<0.05$, $\eta_{\mathrm{p}}{ }^{2}=0.08$. Additionally, the right-left compatibility effect was

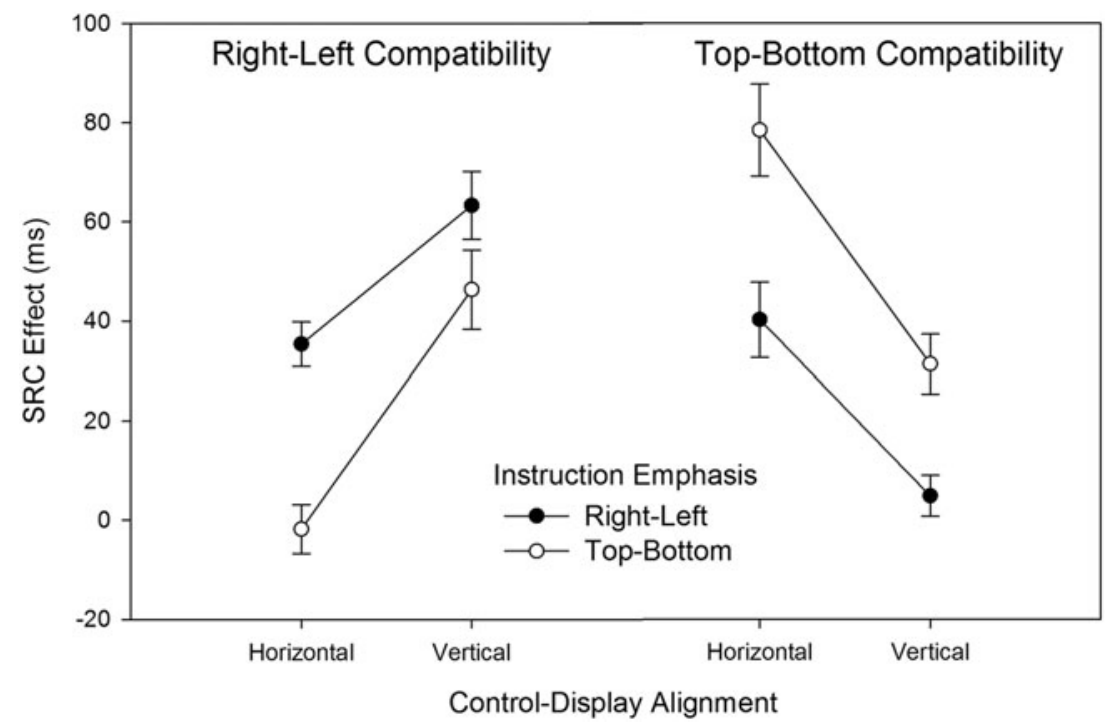

Fig. 4 Right-left and top-bottom RT compatibility effects (incompatible RT - compatible RT) with standard errors for right-left (filled circles) and topbottom (open circles) instructions and horizontal and vertical CDA 
greater with right-left instructions than top-bottom instructions, $F(1,62)=11.08, p<0.05, \eta_{\mathrm{p}}{ }^{2}=0.11$. Instruction type had no effect on PEs by itself, $F(1,62)=1.50, p=0.23, \eta_{\mathrm{p}}{ }^{2}=0.02$, and no other interactions were significant (all $F_{\mathrm{S}}<1.0$, all $p \mathrm{~s}>0.3$ ).

\section{Discussion}

The present study shows that the location of the control panel in relation to the display (CDA) determined the direction of the SRC prevalence effect. When the control panel was positioned above or below the display, right-left compatibility effects were prevalent, and when the control panel was to the right or left of the display, top-bottom compatibility effects were prevalent. We believe that CDA leads to an absolute spatial relation between the stimulus and response sets that changes depending on the panel position-vertical CDA leads to an absolute right-left relation and horizontal CDA leas to an absolute top-down relation. The existence of an absolute spatial relation in one dimension increases the similarity, or dimensional overlap, of stimuli and responses in that dimension, leading to greater salience of that dimension and relatively larger compatibility effects (Kornblum, Hasbroucq, \& Osman, 1990; Vu \& Proctor, 2002).

The current results are at odds with accounts of prevalence effects that rely on the body as a reference frame such as the midline-barrier account (Nicoletti \& Umiltà, 1985) and spatioanatomical account (Nicoletti \& Umiltà, 1984). Regardless of CDA in the current experiment, effector (right and left index fingers were used) and the body midline (participants were always aligned vertically with the response positions) should have consistently elicited right-left prevalence effects. However, the top-bottom prevalence effect with horizontal CDA was larger than the right-left prevalence effect with vertical CDA even though the effectors and midline always emphasized the right-left dimension. Thus, even if the effector type and body midline influenced which task dimension is most salient under some conditions, their influence is negligible compared to the effect of CDA.

Although our results are consistent with an allocentric CDA account, it remains unclear as to whether the effect of CDA is due to the relation between stimulus and response button positions or between stimulus and response effector locations since they were perfectly confounded in the current experiment. For example, when the control panel was aligned below the display, the stimulus locations not only had an absolute right-left spatial relation with the buttons but also with the fingers that were resting on the buttons. Although future work is required to untangle these two relations, either case would remain an issue of alignment between stimulus and response sets rather than effector or body frame of reference alone.

In addition to CDA, Instruction type also interacted with right-left and top-bottom compatibility effects, leading to larger compatibility effects in the dimension matching the instructions. Although SRC effects increased in the dimension emphasized by the instructions, the effect of instruction was only additive with that of CDA, indicating that task instruction alone does not determine the dominant SRC dimension. This finding is in line with prior research by Vu and Proctor (2001) showing that although instructions seem to influence the size of 2-D SRC effects, the right-left prevalence effect is not entirely due to them. Although a particular response dimension can be made more salient by instructions as suggested by Hommel (1993, 1996) and Ansorge and Wuhr (2004), it is separable from CDA in determining of dimensional salience.

Last, there was an interaction between Right-Left Compatibility and Top-Bottom compatibility indicating that the compatibility effect in one dimension was smaller when the other dimension was incompatibly mapped. A similar pattern was found in previous studies of 2-D compatibility effects (Hommel, 1996; Proctor, Van Zandt, Lu, \& Weeks, 1993). Hommel (1996) interpreted this compatibility interaction in terms of logical recoding. In short, when a response strategy is chosen for one S-R dimension, it may also be automatically applied to another S-R dimension (see Hommel, 1996, for more detail). For example, if an upper-left target requires a bottom-right response (both right-left and top-bottom dimensions incompatible), the application of a "respond opposite" strategy for the right-left dimension (respond right to the left) also is applied to the top-down dimension (respond bottom to top). This redundancy leads to an underadditive effect on RTs when both dimensions are incompatible. Of importance to the current study, the interaction between the compatibility in each dimension was independent from CDA, indicating that the influence of CDA also was independent from logical recoding.

Acknowledgments This project was supported in part by NASA cooperative agreement NNX09AU66A: Group 5 URC-Center for Human Factors in Advanced Aeronautics Technologies (Brenda Collins, technical monitor).

\section{References}

Ansorge, U., \& Wuhr, P. (2004). A response-discrimination account of the Simon effect. Journal of Experimental Psychology: Human Perception and Performance, 30, 365-377.

Chan, K. L., \& Chan, A. S. (2011). Spatial stimulus-response compatibility for hand and foot controls with vertical plane visual signals. Displays, 32, 237-243.

Heister, G., Schroeder-Heister, P., \& Ehrenstein, W. H. (1990). Spatial coding and spatioanatomical mapping: Evidence for a hierarchical model of spatial stimulus-response compatibility. In R. W. Proctor \& T. G. Reeve (Eds.), Stimulus-response compatibility: An integrated perspective (pp. 117-143). Amsterdam: North-Holland.

Hommel, B. (1993). Inverting the Simon effect by intention: Determinants of direction and extent of effects of irrelevant spatial information. Psychological Research, 55, 270-279. 
Hommel, B. (1996). No prevalence of right-left over top-bottom spatial codes. Perception \& Psychophysics, 43, 102-110.

Kornblum, S., Hasbroucq, T., \& Osman, A. (1990). Dimensional overlap: Cognitive basis for stimulus-response compatibility: A model and taxonomy. Psychological Review, 97, 253-270.

Ladavas, E., \& Moscovitch, M. (1984). Must egocentric and environmental frames of reference be aligned to produce spatial S-R compatibility effects? Journal of Experimental Psychology: Human Perception and Performance, 10, 205-215.

Lamberts, K., Tavernier, G., \& d'Ydewalle, G. (1992). Effects of multiple reference points in spatial stimulus-response compatibility. Acta Psychologica, 79, 115-130.

Nicoletti, R., \& Umiltà, C. (1984). Right-left prevalence in spatial compatibility. Perception \& Psychophysics, 35, 333-343.

Nicoletti, R., \& Umiltà, C. (1985). Responding with hand and foot: The right/left prevalence in spatial compatibility is still present. Perception and Psychophysics, 38, 211-216.

Nicoletti, R., Anzola, G. P., Luppino, G., Rizzolatti, G., \& Umiltà, C. (1982). Spatial compatibility effects on the same side of the body midline. Journal of Experimental Psychology: Human Perception and Performance, 8, 664-673.

Nicoletti, R., Umiltà, C., Tressoldi, E. P., \& Marzi, C. A. (1988). Why are right-left spatial codes easier to form than above-below ones? Perception \& Psychophysics, 43, 287-292.
Proctor, R. W., \& Reeve, T. G. (Eds.). (1990). Stimulus-response compatibility: An integrated perspective. Amsterdam: North-Holland.

Proctor, R. W., Van Zandt, T., Lu, C. H., \& Weeks, D. J. (1993). Stimulusresponse compatibility for moving stimuli: Perception of affordances or directional coding? Journal of Experimental Psychology: Human Perception and Performance, 19, 81-91.

Roswarski, T. E., \& Proctor, R. W. (1996). Multiple spatial codes and temporal overlap in choice-reaction tasks. Psychological Research, 59, 196-211.

Rubichi, S., Nicoletti, R., Pelosi, A., \& Umiltà, C. A. (2004). Right-left prevalence effect with horizontal and vertical effectors. Perception \& Psychophysics, 2, 255-263.

Vu, K.-P. L., \& Proctor, R. W. (2001). Determinants of right-left and topbottom prevalence for two-dimensional spatial compatibility. Journal of Experimental Psychology: Human Perception and Performance, 27, 813-828.

Vu, K.-P. L., \& Proctor, R. W. (2002). The prevalence effect in twodimensional stimulus-response compatibility is a function of the relative salience of the dimensions. Perception and Psychophysics, 64, 815-828.

Vu, K. P. L., Pellicano, A., \& Proctor, R. W. (2005). No overall right-left prevalence for horizontal. Perception \& Psychophysics, 67, 929938. 